\title{
Retrospective analysis of large-scale research screening of construction workers for the early diagnosis of mesothelioma
}

\author{
TOMOKO HIROHASHI $^{1}$, KIYOKO IGARASHI ${ }^{2}$, MASAAKI ABE $^{1}$, MASAHIRO MAEDA $^{3}$ and OKIO HINO $^{1}$ \\ ${ }^{1}$ Department of Pathology and Oncology, School of Medicine, Juntendo University, Bunkyo-Ku, Tokyo 113-8421; \\ ${ }^{2}$ Byotai-seiri Laboratory, Itabashi-Ku, Tokyo 173-0025; ${ }^{3}$ Immuno-Biological Laboratories, Co., Ltd., \\ Fujioka-Shi, Gunma 375-0005, Japan
}

Received July 1, 2013; Accepted September 23, 2013

DOI: $10.3892 /$ mco.2013.197

\begin{abstract}
The early diagnosis of mesothelioma, an aggressive malignant tumor, is considered to be important for prognosis. $\mathrm{X}$-ray is commonly used for the assessment of a mass in a population exhibiting a risk factor. However, there are currently no available studies indicating that such an assessment may be used to achieve early diagnosis and improve the patient's outcome. We previously reported that $\mathrm{N}-\mathrm{ERC} /$ mesothelin may be a useful blood tumor marker for mesothelioma. In order to investigate whether this tumor marker is useful for early diagnosis in a mass examination, in 2007 we initiated a 5-year large-scale screening of construction workers with a risk of asbestos exposure in Japan. Blood samples were collected annually and $\mathrm{N}-\mathrm{ERC} / \mathrm{mes}$ thelin levels were determined. Based on the results of those findings, along with medical history and related data, we screened the participants to identify a high-risk population. As a result, 62 subjects were identified among $~ 40,000$ participants as the high-risk population. Two of these 62 participants subsequently developed mesothelioma, although the remaining participants have not yet developed mesothelioma. In conclusion, N-ERC/mesothelin may be useful as a blood tumor marker in the early diagnosis of mesothelioma in a mass examination. A future prospective study to confirm the findings of this research screening is currently under planning.
\end{abstract}

\section{Introduction}

Mesothelioma is a relatively rare disease, with $\sim 1,000$ cases of mesothelioma-related mortality annually in Japan (1). However, its incidence has been on the increase and is predicted to reach a peak in 2030 in Japan (2). There is currently no standard diagnostic method for this disease worldwide (3). In

Correspondence to: Professor Okio Hino, Department of Pathology and Oncology, School of Medicine, Juntendo University, 2-1-1 Hongo, Bunkyo-Ku, Tokyo 113-8421, Japan

E-mail: ohino@juntendo.ac.jp

Key words: large-scale research screening, early diagnosis, mesothelioma most countries, pathological confirmation from tumor biopsy samples is used for a confirmatory diagnosis (3). However, a tumor biopsy is typically performed only if this disease is suspected, since it is a highly invasive procedure. Thus, numerous patients with mesothelioma are diagnosed at a late stage. Therefore, the establishment of a more convenient and non-invasive method for the early diagnosis of mesothelioma is a pressing issue. Mesothelioma is highly associated with exposure to asbestos and X-rays are commonly used in a mass examination for subjects with such a risk factor (4). However, there are currently no available studies indicating that an $\mathrm{X}$-ray mass examination may be used for the early diagnosis of mesothelioma (5) to improve patient survival.

We previously reported on the renal carcinoma gene ERC, which is highly expressed in renal cancer in Eker rats (6). We also reported that ERC is a homolog of the human megakaryocyte potentiating factor/mesothelin gene $(7,8)$. The human mesothelin gene product is a $71-\mathrm{kDa}$ precursor protein, which is cleaved by a furin-like protease into a $40-\mathrm{kDa}$ C-termimal fragment that remains membrane-bound and a $31-\mathrm{kDa} \mathrm{N}$-terminal fragment (N-ERC/mesothelin) that is secreted into the bloodstream (9). Therefore, N-ERC/mesothelin would be expected to serve as a specific and easily-measured biomarker of mesothelioma. We developed an enzyme-linked immunosorbent assay (ELISA) system that detects N-ERC/mesothelin (10) and recently reported that $\mathrm{N}-\mathrm{ERC} / \mathrm{mesothelin}$ may be useful for the early diagnosis of mesothelioma (11).

We initiated a 5-year large-scale research screening in 2007, in order to determine whether this blood tumor marker is useful for early diagnosis in a mass examination and to recommend an effective screening method that may be conducted on a large scale. This is an interim retrospective analysis report of this research screening for a future prospective study that is currently under planning.

\section{Materials and methods}

Screening population. Adult male or female participants who were members of the Tokyo General Construction Workers Union and the Tokyo Doken National Health Insurance Association were invited to participate in this research screening, since they are or have been at a risk of asbestos exposure, considering the nature of their occupations, which 
included construction or plumbing. When the participants underwent annual health check-ups, written informed consent was obtained prior to their participation, regardless of their asbestos exposure history or the duration of their construction work history. This being a large-scale screening, the target number of participants was $\sim 40,000$.

Screening method. This research screening was approved by the Institutional Review Board (IRB) of Juntendo University School of Medicine, its Affiliated Hospital (Tokyo, Japan)and the Immuno-Biological Laboratories (Gunma, Japan). This study was conducted at 85 research sites, which were approved by the IRB. Blood samples were collected on an annual basis. The high-risk population was identified based on the results of an annual N-ERC/mesothelin assessment. The participants who were selected as members of the high-risk population were advised to visit Juntendo University or its Affiliated Hospital for further assessments to achieve early diagnosis. As a control population for data analysis, a low-risk population was also selected for comparison with the high-risk population.

Identification of the high-risk population. As previously reported (12), we initially defined the participants as high-risk if they exhibited abnormal levels $(>8.0 \mathrm{ng} / \mathrm{ml})$ of $\mathrm{N}-\mathrm{ERC} / \mathrm{mesothelin}$. However, it was considered that this criterion was not sufficient for a screening and that more optimal criteria were required. We therefore set the new criteria for the high-risk population as follows: i) human anti-mouse antibody (HAMA) not detected; ii) absence of any evidence of renal dysfunction based on medical history and a laboratory test. This criterion was included to consider the effect of renal failure on this marker (13); iii) age $\geq 35$ years; iv) detection of abnormal values of $\mathrm{N}-\mathrm{ERC} /$ mesothelin on more than two occasions during the annual assessments.

The final confirmation of the high-risk population was performed by a case review committee, which was held on a monthly basis.

Data on a history of asbestos exposure were not collected unless the participants developed mesothelioma, since the scope of our research was a mass scale screening of subjects with a risk factor.

Case review committee. This committee was comprised of at least one physician, one data manager, one technician familiar with $\mathrm{N}-\mathrm{ERC} / \mathrm{mes}$ thelin and one administrator from the Tokyo General Construction Workers Union. The role of the committee was to ultimately identify the high-risk population.

Selection of the low-risk population. In order to characterize the high-risk population, a low-risk population was also selected as a control prior to the initiation of data analysis. In total, 7,850 participants from 17 approved research sites were selected as the low-risk population, who underwent $\mathrm{N}-\mathrm{ERC} / \mathrm{mesothelin}$ measurements at least twice and exhibited no abnormal values.

Confirmation of the development of mesothelioma in the high-risk population. The confirmatory diagnosis of mesothelioma was determined via tumor biopsy in the context of appropriate clinical and radiological findings.
Preparation of anti-ERC/mesothelin antibodies. The anti-N-ERC/mesothelin monoclonal antibody (m Ab) clones 7E7 and 16K16 were described previously $(10,11)$. $\mathrm{N}$-ERC/mesothelin, expressed in Escherichia coli as a glutathione-S-transferase and histidine-tagged fusion protein, was purified and used as an immunogen. Splenocytes from immunized mice were fused with the X-73-Ag8.653 mouse myeloma cells. Using ELISA, the supernatants of the hybridoma cells were screened by their reactivity to the immunogen and several positive clones were selected with the limiting dilution method. The 7E7 and 16K16 clones were selected for ELISA.

ELISA. Blood N-ERC/mesothelin levels were determined with the sandwich ELISA system using 7E7 and 16K16 mAbs. The details of this analysis were previously described $(10,11)$. Briefly, microtiter plates (96 wells) were coated with $100 \mu \mathrm{l} /$ well of $100 \mathrm{mmol} / \mathrm{l}$ carbonate buffer $(\mathrm{pH} 9.5)$ containing 7E7 mAb. The plates were washed with phosphate-buffered saline with $0.1 \%$ Tween-20 (PBS-T) and blocked with $200 \mu \mathrm{l} /$ well $1 \%$ (w/v) bovine serum albumin (BSA) in PBS containing $0.05 \% \mathrm{NaN}_{3}$. After washing with PBS-T, 100- $\mu$ l aliquots of test samples or recombinant $\mathrm{N}-\mathrm{ERC} / \mathrm{mes}$ thelin as a standard, serially diluted in PBS-T with $1 \%$ BSA, were added in duplicate to the wells, followed by incubation at $37^{\circ} \mathrm{C}$ for $1 \mathrm{~h}$. After washing with PBS-T, $100 \mu 1$ horseradish peroxidase (HRP)-conjugated $16 \mathrm{~K} 16 \mathrm{mAb}$ was added to each well, followed by incubation for $30 \mathrm{~min}$ at $4^{\circ} \mathrm{C}$. The wells were washed with PBS-T and $100 \mu \mathrm{l}$ of freshly prepared tetramethyl benzidine solution was added as a substrate. The resulting mixture was incubated in the dark for $30 \mathrm{~min}$ at room temperature. The reaction was terminated by the addition of $100 \mu \mathrm{l}$ of $1 \mathrm{~N} \mathrm{H}_{2} \mathrm{SO}_{4}$. The absorbance of the solution was measured at $450 \mathrm{~nm}$ in an ELISA reader (EMax; Molecular Devices Co. Ltd., Sunnyvale, CA, USA). In the case of samples with extra value due to HAMA, they were re-measured with sample diluents containing normal mouse IgG in PBS-T with $1 \%$ BSA as substitute for PBS-T with $1 \%$ BSA.

Statistical analysis. A longitudinal data analysis model was used to estimate the mean change from Time 1 (the first assessment) over time. The model included factors for group, time and group-by-time interaction as fixed effects. An unstructured covariance matrix was used to model the correlation among repeated assessments. Ninety-five percent confidence intervals (95\% CIs) were provided for the difference in change from Time 1 values over time between the high-risk and low-risk groups, using an appropriate contrast of least squares means and referencing a t-distribution. $\mathrm{P}$ values were also computed based on the contrast statistics. A step-down closed testing procedure was employed to test the differences in change from the Time 1 value. The procedure started at Time 5 and continued in a descending order in time until the lower limit of the $95 \%$ CI for difference in change from Time 1 at a particular time point failed to exceed $0 \mathrm{ng} / \mathrm{ml}$. $\mathrm{P}<0.05$ was considered to indicate a statistically significant difference.

\section{Results}

Identification of the high-risk population. As of September 26, 2010, 40,000 participants were enrolled in this research 
Table I. Mean N-ERC/mesothelin (ng/ml) in the low- and high-risk populations by gender and by assessment.

\begin{tabular}{|c|c|c|c|c|c|c|c|c|}
\hline Risk group & Gender & Assessment time & No. & Mean & SD & $\operatorname{Max}$ & Median & Min \\
\hline High & $\mathrm{F}$ & Time 1 & 26 & 9.34 & 3.30 & 19.39 & 9.26 & 3.23 \\
\hline High & $\mathrm{F}$ & Time 2 & 26 & 10.46 & 5.39 & 28.54 & 9.13 & 2.49 \\
\hline High & $\mathrm{F}$ & Time 3 & 15 & 9.90 & 2.40 & 15.81 & 9.90 & 5.23 \\
\hline High & $\mathrm{F}$ & Time 4 & 5 & 11.03 & 1.24 & 12.63 & 10.90 & 9.22 \\
\hline High & M & Time 1 & 36 & 10.17 & 3.80 & 27.95 & 9.25 & 3.99 \\
\hline High & M & Time 2 & 39 & 10.16 & 5.33 & 37.37 & 8.95 & 5.25 \\
\hline High & M & Time 3 & 18 & 12.09 & 6.54 & 31.49 & 10.37 & 6.30 \\
\hline High & M & Time 4 & 12 & 17.40 & 19.57 & 78.60 & 12.35 & 6.80 \\
\hline High & M & Time 5 & 5 & 12.10 & 3.40 & 16.48 & 12.47 & 8.69 \\
\hline Low & $\mathrm{F}$ & Time 1 & 1834 & 2.41 & 1.15 & 7.91 & 2.14 & 0.56 \\
\hline Low & $\mathrm{F}$ & Time 2 & 1842 & 3.05 & 1.43 & 7.95 & 2.76 & 0.05 \\
\hline Low & $\mathrm{F}$ & Time 3 & 753 & 3.44 & 1.49 & 7.92 & 3.12 & 0.52 \\
\hline Low & $\mathrm{F}$ & Time 4 & 220 & 3.62 & 1.53 & 7.88 & 3.42 & 0.48 \\
\hline Low & $\mathrm{F}$ & Time 5 & 37 & 3.82 & 1.60 & 7.32 & 3.84 & 0.05 \\
\hline Low & $\mathrm{M}$ & Time 1 & 5988 & 2.14 & 1.15 & 7.87 & 1.84 & 0.05 \\
\hline Low & $\mathrm{M}$ & Time 2 & 5980 & 2.63 & 1.38 & 7.96 & 2.33 & 0.05 \\
\hline Low & M & Time 3 & 2956 & 2.92 & 1.47 & 7.96 & 2.66 & 0.05 \\
\hline Low & $\mathrm{M}$ & Time 4 & 1107 & 3.15 & 1.44 & 7.96 & 2.92 & 0.05 \\
\hline Low & M & Time 5 & 220 & 2.88 & 1.32 & 7.52 & 2.56 & 0.28 \\
\hline
\end{tabular}

F, female; M, male; SD, standard deviation; Max, maximum; min, minimum.

Table II. Mean N-ERC/mesothelin level and mean change from Time 1 assessment in the low- and high-risk populations (ng/ml).

\begin{tabular}{|c|c|c|c|c|c|}
\hline \multirow[b]{2}{*}{$\begin{array}{l}\text { Assessment } \\
\text { time }\end{array}$} & \multicolumn{2}{|c|}{ Low-risk population } & \multicolumn{2}{|c|}{ High-risk population } & \multirow[b]{2}{*}{$\begin{array}{l}\text { Difference in mean change } \\
\text { from Time } 1(95 \% \mathrm{CI})\end{array}$} \\
\hline & Mean $\pm \mathrm{SE}$ & $\begin{array}{l}\text { Mean change from } \\
\text { Time1 }(95 \% \mathrm{CI})\end{array}$ & Mean \pm SE & $\begin{array}{l}\text { Mean change from } \\
\text { Time } 1(95 \% \mathrm{CI})\end{array}$ & \\
\hline Time 1 & $2.20 \pm 0.01$ & & $9.83 \pm 0.15$ & & \\
\hline Time 2 & $2.73 \pm 0.02$ & $0.53(0.50-0.55)$ & $10.31 \pm 0.19$ & $0.48(0.20-0.76)$ & $-0.05(-0.33,0.23)$ \\
\hline Time 3 & $3.27 \pm 0.02$ & $1.07(1.04-1.11)$ & $13.03 \pm 0.25$ & $3.21(2.80-3.61)$ & $2.13(1.72,2.54)$ \\
\hline Time 4 & $3.83 \pm 0.03$ & 1.63 (1.58-1.69) & $14.80 \pm 0.30$ & $4.98(4.48-5.48)$ & $3.34(2.84,3.85)$ \\
\hline Time 5 & $4.33 \pm 0.06$ & $2.13(2.02-2.25)$ & $17.28 \pm 0.47$ & $7.45(6.62-8.23)$ & $5.32(4.47,6.16)$ \\
\hline
\end{tabular}

SE, standard error; CI, confidence interval.

screening and a total of 124,288 blood samples were collected and analyzed for N-ERC/mesothelin. Among these, 1,603 samples exhibited abnormal values, defined as $>8.00 \mathrm{ng} / \mathrm{ml}$ of $\mathrm{N}-\mathrm{ERC} / \mathrm{mes}$ thelin, which was rarely detected among healthy volunteers $(10,11)$. These samples were re-analyzed, in order to exclude HAMA detection. Following re-analysis, 714 samples still exhibited high values. Based on the decision of the case review committee, 62 participants were finally identified as the high-risk population. According to X-ray findings, there were no reports indicating suspected mesothelioma among the high-risk population in this interim assessment. The mean age of the high-risk subjects was 56 years (range, 35-76 years).

Data analysis of $\mathrm{N}$-ERC/mesothelin in the high-and low-risk population. Mean N-ERC/mesothelin value by risk group, gender and assessment time are presented in Table I. The mean $\mathrm{N}$-ERC/mesothelin level in the low-risk population were $\sim 2.20-4.33 \mathrm{ng} / \mathrm{ml}$, similar to that in healthy volunteers and the mean $\mathrm{N}-\mathrm{ERC} /$ mesothelin level in the high-risk population was 9.83-17.28 ng/ml, similar to that in patients with mesothelioma, as we previously reported (11). No significant differences in the mean N-ERC/mesothelin level by age (data not shown in Table I) or gender were identified between the two populations, although the number of data points in the high-risk population was limited.

We also calculated the mean $\pm \mathrm{SE}$ of $\mathrm{N}$-ERC/mesothelin value and the mean change in $\mathrm{N}-\mathrm{ERC} /$ mesothelin value from the first assessment in the two populations (Table II). In the high-risk population, the level increased by $\sim 2.0$ points annually, whereas it was increasing by only 0.4 points annually in 


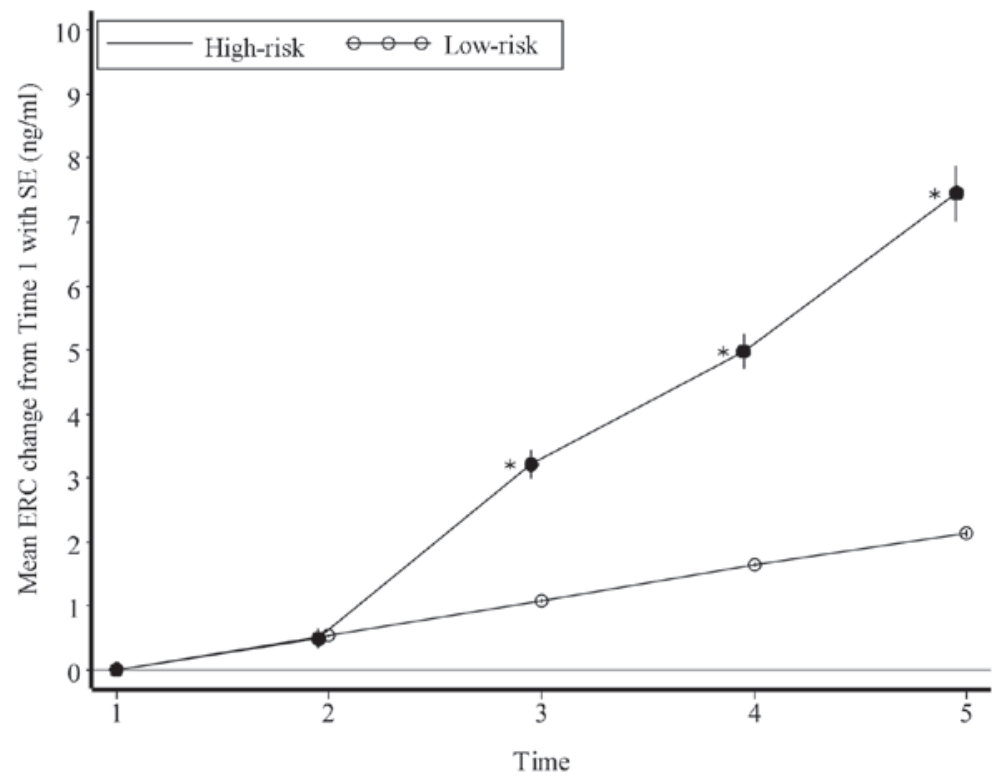

Figure 1. Mean change from assessment 1 (Time 1) in N-ERC/mesothelin (SE). ("P<0.0001, for high-risk vs. low-risk). SE, standard error.

the low-risk population. Therefore, there was a tendency for $\mathrm{N}-\mathrm{ERC} / \mathrm{mesothelin} \mathrm{levels} \mathrm{to} \mathrm{increase} \mathrm{significantly} \mathrm{during} \mathrm{this}$ study period in the high-risk population, compared to those in the low-risk population (Fig. 1). In addition, two participants in the high-risk population ultimately developed mesothelioma. The background information and outcome of the participants who developed mesothelioma is summarized below:

Mesothelioma case 1 . We previously reported a summary of this participant in the form of a case report (13).

Mesothelioma case 2. The participant was a 72-year-old male, with a 50-year history of asbestos exposure. This participant submitted his blood samples for $\mathrm{N}$-ERC/mesothelin analysis annually during the first 3 years and the values were abnormally high on all three measurements $(9.3,8.8$ and $11.1 \mathrm{ng} / \mathrm{ml}$ ). However, since the $\mathrm{N}-\mathrm{ERC} / \mathrm{mes}$ thelin values were stable, the participant did not receive a N-ERC/mesothelin test in the 4th year, although the site staff advised him to receive a test several times. When the patient visited the research site in the 5th year, the N-ERC/mesothelin levels had suddenly escalated to $78.6 \mathrm{ng} / \mathrm{ml}$ and he was diagnosed with epithelioid-type mesothelioma.

Notably, two other participants from the high-risk population developed other types of cancer (lung and appendiceal cancer). Since other cancer types were beyond the scope of this research screening, we were not able to compare the incidence of other types of cancer between the high-risk and the entire populations.

\section{Discussion}

Since the mean N-ERC/mesothelin levels in the high-risk population were similar to those among patients with mesothelioma and two participants from the high-risk population developed mesothelioma, N-ERC/mesothelin levels may be a useful marker for the early diagnosis of this disease in a large-scale setting. We plan to follow up on all the participants of the high-risk population and prospectively assess how many ultimately develop mesothelioma, while continuing to investigate for early signs of mesothelioma. For this purpose, we plan to encourage the high-risk population to visit the hospital annually for a check up.

One participant from the high-risk population failed to undergo the annual N-ERC/mesothelin test and was diagnosed with mesothelioma in the following year. Had the patient not missed this annual test, he may have been diagnosed earlier and may have been a candidate for surgery. Therefore, we strongly recommend encouraging the participants of the high-risk population to receive an additional health check-up at least annually, even if there are no clinical symptoms. The screening system reported herein currently appears to be efficient; however, we hope to provide a more credible screening system after all the analyses are completed.

\section{Acknowledgements}

The authors would like to thank the participants and the investigators, the members of the Tokyo General Construction Workers Union and the Tokyo Doken National Health Insurance Association for their cooperation in the conduction of this study. We would also like to thank Mr Akira Wakana for providing advice on statistical analyses. We are also grateful to the Vehicle Racing Commemorative Foundation and the Ministry of Education, Culture, Sports, Science and Technology of Japan for the financial support of this study (no. 221S0001).

\section{References}

1. Gemba K, Fujimoto N, Kato K, Aoe K, Takeshima Y, Inai K and Kishimoto T: National survey of mesothelioma and asbestos exposure in Japan. Cancer Sci 103: 483-490, 2012.

2. Nakano T: Current therapies for malignant pleural mesothelioma. Environ Health Prev Med 13: 75-83, 2008.

3. Robinson BW, Musk AW and Lake RA: Mesothelioma. Lancet 366: 397-408, 2005.

4. Reid A, de Klerk N, Ambrosini G, Olsen N, Pang SC and Musk AW: The additional risk of mesothelioma in former workers and residents of Wittenoom with benign pleural disease or asbestosis. Occup Environ Med 62: 665-669, 2005. 
5. Sandén A and Järvholm B: A study of possible predictors of mesothelioma in shipyard workers exposed to asbestos. J Occup Med 33: 770-773, 1991

6. Hino O, Kobayashi E, Nishizawa M, et al: Renal carcinogenesis in the Eker rat. J Cancer Res Clin Oncol 121: 602-605, 1995.

7. Yamashita Y, Yokoyama M, Kobayashi E, Takai S and Hino O: Mapping and determination of the cDNA sequence of the Erc gene preferentially expressed in renal cell carcinoma in the Tsc2 gene mutant (Eker) rat model. Biochem Biophys Res Commun 275: 134-140, 2000.

8. Hino O: Multistep renal carcinogenesis in the Eker (Tsc 2 gene mutant) rat model. Curr Mol Med 4: 807-811, 2004.

9. Hassan R, Bera T and Pastan I: Mesothelin: a new target for immunotherapy. Clin Cancer Res 10: 3937-3942, 2004.

10. Shiomi K, Miyamoto H, Segawa T, et al: Novel ELISA system for detection of N-ERC/mesothelin in the sera of mesothelioma patients. Cancer Sci 97: 928-932, 2006.
11. Shiomi K, Hagiwara Y, Sonoue K, et al: Sensitive and specific new enzyme-linked immunosorbent assay for N-ERC/mesothelin increases its potential as a useful serum tumor marker for mesothelioma. Clin Cancer Res 14: 1431-1437, 2008.

12. Imashimizu K, Shiomi K, Maeda M, Aoki N, Igarashi K, Suzuki F, Koizumi M, Suzuki K and Hino O: Feasibility of large-scale screening using N-ERC/mesothelin levels in the blood for the early diagnosis of mesothelioma. Exp Ther Med 2: 409-411, 2011.

13. Shiomi K, Shiomi S, Ishinaga Y, et al: Impact of renal failure on the tumor markers of mesothelioma, N-ERC/mesothelin and osteopontin. Anticancer Res 31: 1427-1430, 2011. 